Original Article

\title{
ARTIFICIAL NEURAL NETWORK AND REGRESSION MODELS TO EVALUATE RHEOLOGICAL PROPERTIES OF SELECTED BRAZILIAN HONEYS
}

\author{
Vanelle M. D. Silva ${ }^{1 \star}$ \\ Wilian S. Lacerda² \\ Jaime V. de Resende 2 \\ 'Universidade Federal de Viçosa, Florestal, Minas Gerais, Brazil \\ ¿Universidade Federal de Lavras, Lavras, Minas Gerais, Brazil \\ *corresponding author: vanelle.silva@gmail.com \\ Received: 12 July 201 9; accepted: 08 May 2020
}

Abstract

The relationships between physico-chemical and rheological properties are considered complex nonlinear systems. Thus, the artificial neural network (ANN) and regression models were used for the rheological characterization of Brazilian honeys, based on lowcost measurements of water content and temperature. The steady shear viscosity ( $\eta$ ) performed well when measured in the test phase in a 2-12-1 neuron multilayer perceptron (MLP) ANN (model 1) with a root mean square error (RMSE) and correlation coefficient (r) equal to 0.0430 and 0.9681 , respectively. The parameter loss modulus ( $\left.G^{\prime \prime}\right)$, storage modulus $\left(G^{\prime}\right)$ and complex viscosity $\left(\eta^{\star}\right)$ were predicted in the temperature sweep test by small amplitude oscillatory shear (SAOS) measurements during heating and cooling, and the MLP ANNs with architectures of 2-9-3 (model 2) and 2-3-3 (model 3) showed RMSE values equal to 0.0261 and 0.0387 in the test phase, respectively. For all the determined parameters, non-linear exponential models showed similar results to models 1, 2 and 3. An ANN with 3-9-3 architecture (model 4) showed RMSE and r for G' equal to 0.0158 and 0.7301 , for $G^{\prime \prime}$ equal to 0.0176 and 0.9581 , and for $\eta^{*}$ equal to 0.0407 and 0.9647 , respectively, in the test phase for date of the frequency sweep test obtained by SAOS. These results were far superior to those obtained by second-order multiple linear models. The acquisition of all models is an important application for the processing of honey and honey-based products, since these properties are essential in engineering calculations and quality control of products.

Keywords: artificial neural network models, Complex Viscosity, loss modulus, regression analysis, Viscosity

\section{INTRODUCTION}

Honey is a fluid food and therefore knowledge of its rheological properties is important in the production processes. This enables better quality control and proper sizing of processing units, since it enables performance evaluation and selection of equipment for product handling, packaging and processing and avoids unnecessary expenditures of beekeepers and the industry (Yanniotis, Skaltsi, \& Karaburnioti, 2006).

In general, honey has a Newtonian behavior, which means the viscosity ( $\eta$ ) of the system is independent of the shear rate applied (Dobre et al., 2012). However, due to the presence of sugar crystals, proteins and other colloids, honey showed a pseudoplastic non-Newtonian behavior in studies by Ahmed et al. (2007), Chen et al. (2009) and Karasu et al. (2015). Moreover, honeys with their viscoelastic properties behave like liquids characteristic of viscous fluids (Ahmed et al., 2007; Chen et al., 2009; Dobre et al., 2012), because in the small amplitude oscillatory shear (SAOS) measurements the $G^{\prime \prime}$ (loss modulus) of honey has a much greater magnitude than that of $G^{\prime}$ (storage modulus). The temperature sweep tests in SAOS measurements are highly sensitive in determining structural changes through heating or by temperature fluctuations 
to which the material may be subjected (Chen et al., 2009; Karasu et al., 2015). Meanwhile, the frequency sweep test shows how the viscoelastic behavior of the material changes with the application rate of the strain amplitude (or tension), where the $G^{\prime \prime}$ (loss modulus) of honey is highly dependent on the angular frequency (Dobre et al., 2012; Kayacier, Yüksel, \& Karaman, 2014).

Normally, the relationships between physico-chemical and rheological properties are considered complex nonlinear systems. Furthermore, the investigation of many of these properties is time-consuming and demands significant laboratory resources (Herrmann et al., 2013). In this context, artificial neural networks (ANN) have the potential to be applied in the estimation and prediction of these properties in foods based on simple and low-cost measures. This is possible due to their ability to learn and generalize the behavior of any complex and non-linear process (Zuluaga-Domínguez, NietoVeloza, \& Quicazán-de-Cuenca, 2017).

ANNs are computational models inspired by the functioning of the human brain and based on the mechanisms of memorization and learning. This tool can learn the relationship between inputs and outputs based on training data. It is therefore widely used for modeling, prediction and classification since it permits the identification of relationships present in limited, incomplete, noisy, dynamic and nonlinear data sources (Mohanraj, Jayaraj, \& Muraleedharan, 2012).

The combined effect of temperature, moisture content and strain rate on viscosity of honeys from Jordan (Al-Mahasneh, Rababah, \& Ma'Abreh, 2013) and honeys from Iran (Ramzi et al., 2015) was evaluated using ANNs with showed good results. The ANN was less accurate than the Adaptive Neuro - Fuzzy Inference System (ANFIS) in modeling the complex viscosity $\left(\eta^{\star}\right)$ of Turkish honeys dependent on the variety, temperature and angular frequency (Kayacier, Yüksel, \& Karaman, 2014). The influence of temperature, frequency and water content on the viscoelastic parameters of honeys from Spain was also evaluated using ANNs and the ANFIS.
In this case, a Multilayer Perceptron (MLP) ANN presented the best predictive ability of all viscoelastic properties (Oroian, 2015). In all studies, the temperature range and number of honey samples were relatively limited considering the wide variety of honeys available and the range of temperatures that can be employed during processing. Furthermore, there are no reports in literature on the use of ANNs for estimating viscoelastic properties by means of temperature scans during heating and cooling.

Thus, the potential use of ANNs to predict the rheological properties of honeys from low-cost measures using larger temperature and water content ranges will expand the application of the obtained network. The present study therefore sought to develop different ANN models to predict viscosity from steady shear measurements, $G^{\prime}, G^{\prime \prime}$ and $\eta^{*}$ from temperature scans during heating, $G^{\prime}, G^{\prime \prime}$ and $\eta^{\star}$ from temperature scans during cooling, where both models are a function of water content and temperature and finally $G^{\prime}, G^{\prime \prime}$ and $\eta^{*}$ from the frequency sweeps, in function of the water content, temperature and angular frequency. Furthermore, models 1 , 2 and 3 were compared to exponential models and model 4 to multiple linear regression models.

\section{MATERIAL AND METHODS}

\section{Rheological data collection}

The data were obtained by Silva et al. (2016) and Silva, Torres Filho, \& Resende (2018), who analyzed twenty-two samples of monofloral honey Assa-peixe (Vernonia polysphaera) (3), Cipó-uva (Serjania lethalis) (5), Eucalyptus (Eucalyptus spp.) (7), and Orange blossom (Citrus sinensis) (7) from the southeast and eighteen samples of multifloral honey from the southeast (8), south (3), northeast (3) and central-west (4) regions of Brazil.

\section{Artificial neural network modeling}

In this study, a supervised multilayer perceptron feedforward artificial neural network (MLP ANN) was used for the development of four independent neural models for the rheological parameter of honeys. Model 1 was developed 


\section{Ј. APPC. SCLI. VOL. 64 N NO. 2 2020}

based on data of $\eta$ determined in steady shear rheological measurements. Models 2 and 3 were developed from data of $G^{\prime}, G^{\prime \prime}$ and $\eta^{*}$ measured in oscillatory tests of temperature scans during heating and cooling, respectively. Model 4 was developed from data of $G^{\prime}, G^{\prime \prime}$ and $\eta^{\star}$ measured in oscillatory tests of frequency scans. For models 1, 2 and 3 the input layers were composed of the variables temperature and water content, while for model 4 the input layers were composed of the variables temperature, water content and angular frequency. The input nodes and the neurons in the output layer for each model are shown in Tab. 1 as the statistical indexes of the inputs and outputs of training and testing data in this study.

For the training phase $75 \%$ of the data was used and $25 \%$ was used in the test phase of the MLP (Faria et al., 2015). The data was normalized to fall within the range of the sigmoidal activation function and the hyperbolic tangent activation function (Khanlari et al., 2012; Xi et al., 2013). The learning algorithm used was backpropagation with momentum which permits the correction of synaptic weights during training based on the difference (error) between the desired value and that calculated by the network (Abbasi-tarighat, Shahbazi, \& Niknam, 2013), and allows that the convergence of weights results in an overall minimum rather than a local minimum of the error (Goktepe et al., 2008).

For analysis of the different network topologies, the activation function (hyperbolic tangent and sigmoidal) and the number of neurons in the

Table 1.

Statistical Indexes of Input and Output data in the training and test process of Multilayer Perceptron Feedforward Neural Network

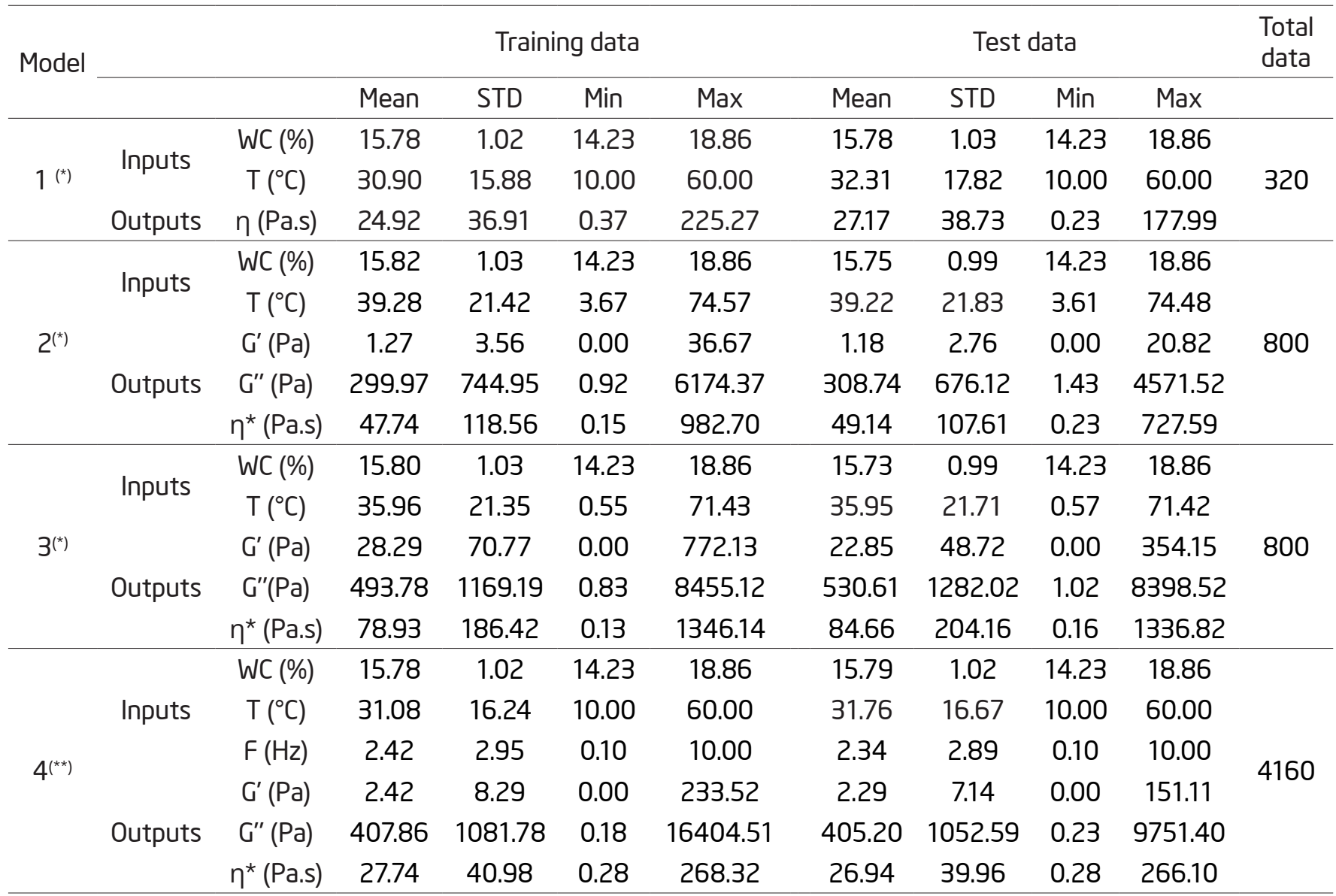

WC: water content; T: temperature; $\eta$ : steady shear viscosity; G': Storage moduli; $G^{\prime \prime}$ : loss moduli; $\eta^{\star}$ : complex viscosity; F: Frequency. Reference: $\left({ }^{\star}\right)$ Silva et al (2016) and $\left({ }^{\star *}\right)$ Silva et al. (2018). 
hidden layer (3, 6, 9, 12 and 15) were varied, and the learning rate to 0.2 and momentum equal to 0.7 were fixed. After obtaining the best topology, different learning rates $(0.2,0.4,0.6)$ and momentum values were tested $(0.3,0.5,0.7$, 0.9) (Llave, Hagiwara, \& Sakiyama, 2012).

The study for the determination of the optimal number of iterations for network training was performed through error convergence analysis (RMSE - Equation 1) for a minimum value of the test data (Phimolsiripol, Siripatrawan, \& Cleland, 2011). Definition of the optimal number of iterations used during training is an essential criterion to avoid overfitting (Linder \& Po, 2003). Selection of the optimal network for each model was determined by a trial and error technique, evaluating different scenarios and configurations and opting for topologies that had lower error values (RMSE). In addition to this statistical criterion, the correlation coefficient ( $\Gamma$ ) was used as a performance measure of the analyzed networks.

The neural network structures were generated using the 2.92 version of Neuroph Neural Network freeware.

\section{Regression Models}

In order to accurately evaluate the predicting technique, the combined effect of water content and temperature on the rheological parameters of the honeys, the same data subsets (training set) for the models 1, 2 and 3, shown in Tab. 1, were used to adjust the following exponential model (Oroian 2012) (Eq.1):

$$
y=a \exp ^{(-b w-c T)}
$$

where $y$ is the predicted response ( $\eta$ (Pa.s), $\eta^{*}$ $(\mathrm{Pa} . \mathrm{s}), \mathrm{G}^{\prime}(\mathrm{Pa})$ or $\left.\mathrm{G}^{\prime \prime}(\mathrm{Pa})\right)$, w is the water content $(\%), T$ is temperature $\left({ }^{\circ} \mathrm{C}\right)$, and $\mathrm{a}$, $\mathrm{b}$, and $\mathrm{c}$ are empirical constants of the models.

A nonlinear regression technique that incorporates the Levenberg-Marquardt method to solve nonlinear regression was used.

The training data for model 4 was further analyzed using multiple regressions and a second order polynomial model expressed in Eq.2: (i< j) (2)

where $y$ is the predicted response $\left(\eta^{*}\right.$ (Pa.s), $G^{\prime}$ $(\mathrm{Pa})$ or $\left.\mathrm{G}^{\prime \prime}(\mathrm{Pa})\right), \beta_{0}$ is the intercept coefficient, $\beta_{i}$ is the linear coefficient, $\beta_{i i}$ is the quadratic coefficient, $\beta_{i j}$ and $\beta_{i j k}$ are interaction coefficients, $X_{i}$ is the water content (\%), $X_{\text {is }}$ is the temperature $\left({ }^{\circ} \mathrm{C}\right)$ and $\mathrm{X}_{k}$ is the frequency $(\mathrm{Hz})$.

A one-factor analysis of variance (ANOVA) was carried out to assess this model. The significances of the coefficients were examined using the t-test.

Performance of the exponential and multiple regression models was evaluated by means of the RMSE and r statistical parameters.

This statistical analysis was performed using version 9.1 of the SAS (Statistical Analysis System) software.

\section{RESULTS}

\section{Performance of the ANNs and regression models}

The optimal number of iterations was obtained by means of the error convergence analysis (Linder \& Po, 2003; Phimolsiripol, Siripatrawan, \& Cleland, 2011) (Fig. 1). The activation function that presented the best results for all models was sigmoidal. This function remains finite even with $x$ values close to $\pm \infty$, which results in more efficient training of the network (Razmi-Rad et al., 2007).

The optimal number of iterations is equal to 100 in training the network with 2-12-1 neurons in its layers, referring to model 1 (Fig. 1-a). From this point a considerable increase in the RMSE test was observed, characterizing the overfitting phenomenon. The network with 2-9-3 neurons in its layers, referring to model 2, showed the lowest test RMSE value when completing 1000 iterations (Fig. 1-b), while for the network 2-3-3, referring to model 3, 2500 


\section{J. APIC. SCLI. VOL. 64 NI. 2 2020}

iterations were required (Fig. 1-c). In both cases a slight increase in the test RMSE was observed after the convergence point. Model 4, whose architecture consisted of 3-9-3 neurons in its layers, required only ten iterations to reach the minimum test RMSE value (Fig. 1-d).
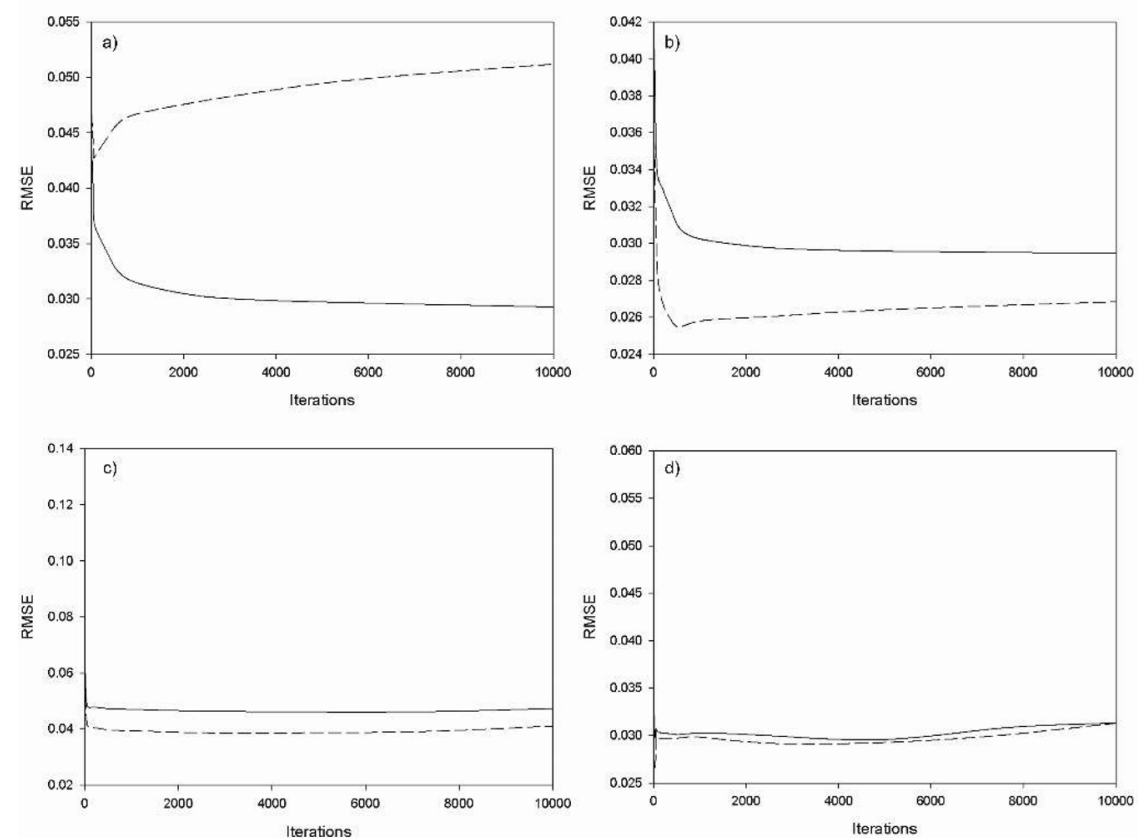

Fig. 1. RMSE of training (solid line) and test (dotted line) sets versus number of iterations for optimum MLP ANN: a) model 1; b) model 2; c) model 3; d) model 4.

After selecting the best activation function and number of neurons in the hidden layer, all model were evaluated with regards to the influence of the learning rate and momentum on their performance. In all models the learning rate value for the best performance was equal to 0.2. This result is in concordance with that reported in literature, considering that low-learning rate values prevent large fluctuations in error and consequent instability problems (Al-Mahasneh, Rababah, \& Ma'Abreh, 2013; Rai, Majumdar, \& Dasgupta, 2005; Rossi et al., 2014). In relation to the term momentum, models 1 and 4 performed better for the value of 0.3 , while models 2 and 3 performed better at the value of 0.7 .

For model 1 (2-12-1 architecture), 500 iterations were required for training. This MLP-ANN produced good results for predicting the viscosity of honey as a function of water content and temperature. The RMSE of the training phase of this model was equal to 0.0359 , while the RMSE of the test phase was equal to 0.0430. Comparing the data calculated by the network and that obtained experimentally, they showed high correlation coefficients for both training and test data, equal to 0.9760 and 0.9681 , respectively.

The exponential model for assessing the combined effect of water content and temperature on viscosity showed higher accuracy than the MLP ANN of model 1 due to the higher coefficient of determination and lower RMSE for the training data, equal to 0.9981 and 0.0101, respectively (Tab. 2). With respect to the test data, both models showed the same performance (Tab. 2) and indicated a reduction in their prediction quality when unknown data was used.

In relation to models 2 and 3 , the error convergence point was reached after 500 and 2500 iterations, respectively. Model 2 (2-9-3 architecture) showed good results in the determination of the rheological properties $G^{\prime}, G^{\prime \prime}$ and $\eta^{*}$ from the water content and temperature in the temperature sweep curves by means of SAOS measurements during heating. This model presented a RMSE test value equal to 0.0255 , while that of training was 0.0310 . When comparing each predicted variable separately in this model with the desired experimental values, the $G^{\prime}$ property was observed to have the highest RMSE training values, equal to 0.0338 , and test values equal to 0.0261 (Tab. 2), as well as the lowest correlation coefficient values, equal to 0.9398 in training and 0.9390 in testing. The properties $G^{\prime \prime}$ and $\eta^{*}$ showed RMSE and training correlation coefficient values equal to 0.0296 and 0.9704 , and 0.0295 and 0.9705 , respectively, and test values of 0.0252 and 0.9731 (Tab. 2).

Model 3 (2-3-3 architecture) also showed good results in the determination of the rheological properties $G^{\prime \prime}$ and $\eta^{*}$ from the water content 
Table 2 .

RMSE and correlation coefficient ( $\ulcorner$ ) of models 1, 2 and 3 variables from the development and test process of a nonlinear exponential and of models 1, 2, 3 and 4 from the best ANNs models

\begin{tabular}{|c|c|c|c|c|c|c|c|c|c|c|c|c|}
\hline \multirow[t]{2}{*}{ Model } & \multirow[t]{2}{*}{$\begin{array}{l}\text { Estimated } \\
\text { variable }\end{array}$} & \multicolumn{3}{|c|}{ Empirical constants ${ }^{1}$} & \multicolumn{2}{|c|}{$\begin{array}{l}\text { Exponential } \\
\text { Model } \\
\text { (Training data) }\end{array}$} & \multicolumn{2}{|c|}{$\begin{array}{l}\text { Exponential } \\
\text { Model } \\
\text { (Test data) }\end{array}$} & \multicolumn{2}{|c|}{ ANN (Training) } & \multicolumn{2}{|c|}{ ANN (Test) } \\
\hline & & A & B & C & RMSE & $r$ & RMSE & $r$ & RMSE & r & RMSE & r \\
\hline 1 & $\eta(P a . s)$ & 0.799 & 2.036 & 6.415 & 0.0101 & 0.9981 & 0.0433 & 0.9700 & 0.0359 & 0.9760 & 0.0430 & 0.9681 \\
\hline \multirow{3}{*}{2} & $\mathrm{G}^{\prime}(\mathrm{Pa})$ & 0.805 & 3.302 & 14.696 & 0.0343 & 0.9356 & 0.0290 & 0.9293 & 0.0338 & 0.9398 & 0.0261 & 0.9390 \\
\hline & $\mathrm{G}^{\prime \prime}(\mathrm{Pa})$ & 0.881 & 2.549 & 10.953 & 0.0282 & 0.9725 & 0.0272 & 0.9688 & 0.0296 & 0.9704 & 0.0252 & 0.9731 \\
\hline & $\eta^{*}($ Pa.s $)$ & 0.881 & 2.549 & 10.953 & 0.0282 & 0.9725 & 0.0272 & 0.9688 & 0.0295 & 0.9705 & 0.0252 & 0.9731 \\
\hline \multirow{3}{*}{3} & $\mathrm{G}^{\prime}(\mathrm{Pa})$ & 0.489 & 2.628 & 8.542 & 0.0659 & 0.6948 & 0.0521 & 0.7055 & 0.0675 & 0.6969 & 0.0486 & 0.6629 \\
\hline & $\mathrm{G}^{\prime \prime}(\mathrm{Pa})$ & 0.911 & 1.684 & 12.549 & 0.0313 & 0.9742 & 0.0320 & 0.9779 & 0.0308 & 0.9758 & 0.0326 & 0.9794 \\
\hline & $\eta^{\star}$ (Pa.s) & 0.913 & 1.687 & 12.526 & 0.0314 & 0.9742 & 0.0320 & 0.9779 & 0.0309 & 0.9759 & 0.0326 & 0.9794 \\
\hline \multirow{3}{*}{4} & $\mathrm{G}^{\prime}(\mathrm{Pa})$ & - & - & - & - & - & - & - & 0.0260 & 0.7244 & 0.0158 & 0.7301 \\
\hline & $\mathrm{G}^{\prime \prime}(\mathrm{Pa})$ & - & - & - & - & - & - & - & 0.0195 & 0.9604 & 0.0176 & 0.9581 \\
\hline & $\eta^{\star}$ (Pa.s) & - & - & - & - & - & - & - & 0.0420 & 0.9636 & 0.0406 & 0.9647 \\
\hline
\end{tabular}

(1)Empirical constants of the exponential model using the training data.

and temperature in the temperature sweep curves by means of SAOS measurements during cooling. However, its statistical parameters were inferior to model 2, where the training RMSE is equal to 0.0464 and that of testing is equal to 0.0387. For each variable separately, the RMSE (Tab. 2) and correlation coefficient were, respectively, 0.0675 and 0.6969 in training, and 0.0486 and 0.6629 in testing for $G$ ', were 0.0308 and 0.9758 in training and 0.0326 and 0.9794 in testing for $G^{\prime \prime}$, and finally were 0.0309 and 0.9759 in training and 0.0326 and 0.9794 in testing for $\eta^{*}$.

Regarding the combined effect of temperature and water content on the viscoelastic properties $\left(G^{\prime}, G^{\prime \prime}\right.$ and $\left.\eta^{\star}\right)$ during temperature scanning, in both heating and cooling, the exponential model showed the same performance as models 2 and 3 with the MLP ANNs, due to the similarity of the obtained statistical parameters (Tab. 2).

Model 4 (3-9-3) presented a training RMSE value equal to 0.0306 , while that of testing was 0.0270 after fifty iterations. The RMSE values (Tab. 2) and correlation coefficients in training were equal to 0.0260 and 0.7244 for $G^{\prime}, 0.0195$ and 0.9604 for $G^{\prime \prime}$ and 0.0420 and 0.9636 for $\eta^{*}$, respectively. In the test, for $G^{\prime}$ they were equal to 0.0158 and 0.7301 , for $G$ " were equal

Table 3.

RMSE and correlation coefficient ( $\ulcorner$ ) of model 4 variables from the development and test process of a multiple-second order polynomial regression

\begin{tabular}{|c|c|c|c|c|c|c|c|c|c|c|c|c|c|c|c|}
\hline \multirow{2}{*}{ Variable } & \multicolumn{11}{|c|}{ Model Coefficient ${ }^{1}$} & \multicolumn{2}{|c|}{ Training data } & \multicolumn{2}{|c|}{ Test data } \\
\hline & $\beta_{0}$ & $\beta_{1}$ & $\beta_{2}$ & $\beta_{3}$ & $\beta_{12}$ & $\beta_{13}$ & $\beta_{23}$ & $\beta_{123}$ & $\beta_{11}$ & $\beta_{22}$ & $\beta_{33}$ & RMSE & $r$ & RMSE & $r$ \\
\hline & - & 0.01 & -0.04 & 0.10 & -0.02 & -0.17 & -0.17 & 0.26 & - & 0.05 & 0.06 & 0.0295 & 6189 & & 0.6932 \\
\hline $\mathrm{G}^{\prime \prime}(\mathrm{Pa})$ & 0.03 & -0.03 & -0.20 & 0.38 & - & -0.40 & -0.51 & 0.56 & 0.04 & 0.20 & - & & & & \\
\hline$\eta^{\star}($ Pa.s) & 0.49 & -0.53 & -1.29 & - & 0.52 & - & - & - & 0.16 & 0.81 & - & 0.0680 & 0.8964 & 0.0640 & 0.9018 \\
\hline
\end{tabular}

(1)Coefficients of the multiple regression model using the training data of model 4.

( - ) p-value > 0.05 obtained by ANOVA. 


\section{Ј. APPC. SCLI. VOL. 64 N NO. 2 2020}

to 0.0176 and 0.9581 and for $\eta^{*}$ were equal to 0.0407 and 0.9647 , respectively. These values of the statistical parameters show a good quality prediction of the properties $G^{\prime \prime}$ and $\eta^{*}$ in function of frequency, water content and temperature by means of the SAOS measurements. Second order multiple regression models were determined to estimate the rheological properties $G^{\prime}, G^{\prime \prime}$ and $\eta^{\star}$ of model 4 (Tab. 3). The prediction quality, based on the correlation coefficient and RMSE parameters, were much lower than the MLP ANN of model 4 (Tab. 2).

\section{DISCUSSION}

The ANNs and exponential nonlinear regression models developed in this study presented good qualities in predicting the viscosity from steady shear rheological measurements (model 1), as well as the properties $G^{\prime}, G^{\prime \prime}$ and $\eta^{*}$ in temperature scans during heating (model 2 ) and $G^{\prime \prime}$ and $\eta^{*}$ in cooling temperature scans (model 3 ) from SAOS measurements. This was possible even when using data unknown by the network or not used in determining the model parameters. In predicting the volume of eggs using a machine vision technique, the authors considered that a mathematical model in literature showed better performance than the ANN (Soltani, Omid, \& Alimardani, 2014), which corroborates with the present study. In contrast, other studies have shown that some mathematical models are less accurate than the ANN (Celekli et al., 2012).

When using the MLP-ANN, similar correlation coefficient values were obtained in the study for predicting the viscosity of fruit juices clarified in the function of temperature and concentration (Rai, Majumdar, \& Dasgupta, 2005) as well as the rheological properties: $\mathrm{K}$ (consistency index), $\mathrm{n}$ (flow behavior index) and $\tau_{0}$ (initial yield stress) as a function of composition (Herrmann et al., 2013). In predicting the viscosity of wild honeys from Jordan as a function of temperature, water content and strain rate, coefficients of determination equal to 0.970 and 0.962 were calculated for the training and test data, respectively, for a network with an architecture of 3-10-1 (AlMahasneh, Rababah, \& Ma'Abreh, 2013). On the other hand, in a study performed with honey from Iran including the prediction of viscosity in function of temperature, water content and strain rate, a correlation coefficient of 0.997 was obtained using an ANN with a genetic algorithm such as the learning algorithm (Ramzi et al., 2015).

The quality of the lower prediction observed for $G^{\prime}$ in models 2 and 3 indicates that its relationships with the input variables are very complex and nonlinear. The honeys presented liquid-like behavior, since $G$ " is much larger than $\mathrm{G}^{\prime}$ in the entire temperature range studied. This is due to a weak interaction without formation of networks between the honey molecules (Ahmed et al., 2007). Thus, Nayik, Dar, \& Nanda (2015) and Silva et al. (2016) studied the effect of temperature on rheological properties of honey only in terms of $G^{\prime \prime}$, because it is a more informative parameter for this type of material. These temperature sweep curves are very important in the study of the effect of temperature fluctuations on the quality of honey observed by hysteresis between the heating and cooling curves (Chen et al., 2009; Karasu et al., 2015; Silva et al., 2016), thereby a proper prediction of the parameters $G^{\prime \prime}$ and $\eta^{*}$ in the function of water content and temperature represents an important practical application.

The property G' showed a low correlation coefficient value despite the lower RMSE, considering that as noted by other authors the temperature and water content do not greatly influence this property (Nayik, Dar, \& Nanda, 2015). When Oroian, (2015) studyied the influence of temperature, frequency and water content on the viscoelastic properties of Spanish honeys with ANN and ANFIS, the MLP ANN defined for the parameter $G$ ' also showed the worst prediction results, with coefficients of determination equal to 0.994 in training, 0.188 in the cross-test and 0.914 in testing. An excellent prediction quality was obtained for the parameters $G^{\prime \prime}$ and $\eta^{*}$, with coefficients of determination greater than 0.993, higher than those of the present study. This improved prediction is associated with greater homogeneity of the data, since the water content range used was from 16.04 to 
$17.82 \%$ and temperature from 5 to $40^{\circ} \mathrm{C}$, more stringent than those of the present study. A better quality of the statistical parameters was also observed in the study when predicting the complex viscosity of honeys from Turkey (Kayacier, Yüksel, \& Karaman, 2014), with an R2 greater than 0.985 . Similarly, the database used presented great homogeneity, considering that the water content ranged from 19.1 to $19.4 \%$ and temperature range used was from 10 to $20^{\circ} \mathrm{C}$. Dieulot \& Skurtys (2013) noted a lack of accuracy when predicting the mechanical behavior of starch films using the complete database. However, after the authors divided this database into three more homogeneous classes via a cluster analysis, prediction of the mechanical properties of each class was more than adequate with higher $R^{2}$ values and lower error values.

Regarding determination of the properties $G^{\prime}, G^{\prime \prime}$ and $\eta^{*}$ in frequency sweeps from the SAOS measurements (model 4), the ANN model presented higher accuracy compared to the second-order polynomial multiple regression model. This demonstrated the effectiveness of the MLP ANN of model 4 when used in non-linear systems with complex relationships between input and output variables.

As in this study, several authors, have compared ANNs with multiple linear regression models and observed the superiority of ANNs for the prediction of the desired parameters due to the non-linearity and complex relationships of the systems, thus indicating their use (Ajala \& Betiku, 2015; Dahmoune et al., 2015; Rahman et al., 2012).

Regarding the determined statistical parameters, with the exception of the parameter $G^{\prime}$ of models 3 and 4, all models were properly trained due to the low RSME value. They also presented good generalization capacity, considering that they provided coherent answers with a low error from data that was not used in the training phase. All properties were determined from simple measurements over wide ranges of temperature and water content values, which represent an important practical application for processing honey and honey-based products, since that these determinations require the use of high-cost equipment and are essential in engineering calculations, quality control and shelf life of the products.

\section{ACKNOWLEDGMENTS}

The authors are grateful for the financial support from the Conselho Nacional de desenvolvimento científico e tecnológico (CNPQ).

\section{REFERENCES}

Abbasi-tarighat, M., Shahbazi, E., \& Niknam, K. (2013). Simultaneous determination of $\mathrm{Mn}^{2+}$ and $\mathrm{Fe}^{3+}$ as complexes in some foods, vegetable and water samples by artificial neural networks. Food Chemistry, 138(2-3), 991-997. http://doi. org/10.1016/j.foodchem.2012.09.099

Ahmed, J., Prabhu, S. T., Raghavan, G. S. V., \& Ngadi, M. (2007). Physico-chemical, rheological, calorimetric and dielectric behavior of selected Indian honey. Journal of Food Engineering, 79, 1207-1213. http:// doi.org/10.1016/j.jfoodeng.2006.04.048

Ajala, S. O., \& Betiku, E. (2015). Yellow Oleander Seed Oil Extraction Modeling and Process Parameters Optimization: Performance Evaluation of Artificial Neural NetworkandResponse Surface Methodology. Journal of Food Processing and Preservation, 39, 1466-1474. http://doi.org/10.1111/jfpp.12366

Al-Mahasneh, M. A., Rababah, T. M., \& Ma'Abreh, A. S. (2013). Evaluating the combined effect of temperature, shear rate and water content on wildflower honey viscosity using adaptive neural fuzzy inference system and artificial neural networks. Journal of Food Process Engineering, 36, 510-520. http://doi.org/10.1111/jppe.12014

Celekli, A., Birecikligil, S. S., Geyik, F., \& Bozkurt, H. (2012). Prediction of removal efficiency of Lanaset Red G on walnut husk using artificial neural network model. Bioresource Technology, 103, 64-70. http:// doi.org/10.1016/j.biortech.2011.09.106

Chen, Y. W., Lin, C. H., Wu, F. Y., \& Chen, H. H. (2009). 
Rheological properties of crystallized honey prepared by a new type of nuclei. Journal of Food Process Engineering, 32(2009), 512-527. http://doi. org/10.1111/j.1745-4530.2007.00227.x

Dahmoune, F., Remini, H., Dairi, S., Aoun, O., Moussi, K., Bouaoudia-Madi, N., ... Madani, K. (2015). Ultrasound assisted extraction of phenolic compounds from P. lentiscus L. leaves: Comparative study of artificial neural network (ANN) versus degree of experiment for prediction ability of phenolic compounds recovery. Industrial Crops and Products, 77,251-261. http://doi.org/10.1016/j.indcrop.2015.08.062

Dobre, I., Georgescu, L. A., Alexe, P., Escuredo, O., \& Seijo, M. C. (2012). Rheological behavior of different honey types from Romania. Food Research International, 49(1), 126-132. http://doi.org/10.1016/j. foodres.2012.08.009

Faria, S., Alberto, C., Anjos, R., Nunes, R., Maria, R. (2015). Evaluation of extra virgin olive oil stability by artificial neural network. Food Chemistry, 179, 3543. http://doi.org/10.1016/j.foodchem.2015.01.100

Goktepe, A. B., Altun, S., Altintas, G., \& Tan, O. (2008). Shear strength estimation of plastic clays with statistical and neural approaches. Building and Environment, 43, 849-860. http://doi.org/10.1016/j. buildenv.2007.01.022

Herrmann, J., Brito, A., Trembley, J., \& Grupa, U. (2013). Development of a rheological prediction model for food suspensions and emulsions. Journal of Food Engineering, 175(4), 481-485. http://doi. org/10.1016/j.jfoodeng.2012.05.034

Karasu, S., Toker, S. O., Yilmaz, M. T., Karaman, S., Dertli, E. (2015). Thermal loop test to determine structural changes and thermal stability of creamed honey: Rheological characterization. Journal of Food Engineering, 150(150), 90-98. http://doi. org/10.1016/j.jfoodeng.2014.10.004 0260-8774/?

Kayacier, A., Yüksel, F., \& Karaman, S. (2014). Dynamic mechanical spectra of selected Turkish honeys: Construction of predictive models for complex viscosity using two different nonlinear modeling techniques. International Journal of Food Properties, 17June 2011), 93-110. http://doi.org/10.1080/1094 2912.2011.614985

Khanlari, G. R., Heidari, M., Momeni, A. A., \& Abdilor, Y. (2012). Prediction of shear strength parameters of soils using artificial neural networks and multivariate regression methods. Engineering Geology, 137-132, 11-18. http://doi.org/10.1016/j.enggeo.2011.12.006

Linder, R., \& Po, S. J. (2003). A new neural network approach classifies olfactory signals with high accuracy. Food Quality and Preference, 14, 435-440. http://doi.org/10.1016/S0950-3293(03)00016-8

Llave, Y. A., Hagiwara, T., \& Sakiyama, T.(2012). Artificial neural network model for prediction of cold spot temperature in retort sterilization of starch-based foods. Journal of Food Engineering, 109(3), 553560. http://doi.org/10.1016/j.jfoodeng.2011.10.024

Mohanraj, M., Jayaraj, S., \& Muraleedharan, C. (2012). Applications of artificial neural networks for refrigeration, air-conditioning and heat pump systems - A review. Renewable and Sustainable Energy Reviews, 16(2), 1340-1358. http://doi. org/10.1016/j.rser.2011.10.015

Nayik, G. A., Dar, B. N., \& Nanda, V. (2015). Physicochemical, rheological and sugar profile of different honeys from Kashmir Valley of India. Arabian Journal of Chemistry, Avaible on. http://doi.org/10.1016/j. arabjc.2015.08.017

Oroian, M. (2012). Physicochemical and rheological properties of Romanian honeys. Food Biophysics, 7. 296-307. http://doi.org/10.1007/s11483-012$9268-x$

Oroian, M. (2015). Influence of temperature, frequency and moisture content on honey viscoelastic parameters - Neural networks and adaptive neuro-fuzzy inference system prediction. LWT - Food Science and Technology, 63(2), 13091316. http://doi.org/10.1016/j.lwt.2015.04.051

Phimolsiripol, Y., Siripatrawan, U., \& Cleland, D. J. (2011). Weight loss of frozen bread dough under isothermal 


\section{_ SllVH ET HL. _ Prediction of rheological properties of honeys}

and fluctuating temperature storage conditions. Journal of Food Engineering, 106(2), 134-143. http:// doi.org/10.1016/j.jfoodeng.2011.04.020

Rahman, M. S., Rashid, M. M., \& Hussain, M. A. (2012). Thermal conductivity prediction of foods by Neural Network and Fuzzy (ANFIS) modeling techniques. Food and Bioproducts Processing, 90(2), 333-340. http://doi.org/10.1016/j.fbp.2011.07.001

Rai, P., Majumdar, G. C., \& Dasgupta, S. (2005). Prediction of the viscosity of clarified fruit juice using artificial neural network: a combined effect of concentration and temperature. Journal of Food Engineering, 68, 527-533. http://doi.org/10.1016/j. jfoodeng.2004.07.003

Ramzi, M., Kashaninejad, M., Salehi, F., Sadeghi Mahoonak, A. R., Ali Razavi, S. M. (2015). Modeling of rheological behavior of honey using genetic algorithm-artificial neural network and adaptive neuro-fuzzy inference system. Food Bioscience, 9, 60-67. http://doi.org/10.1016/j.fbio.2014.12.001

Razmi-Rad, E., Ghanbarzadeh, B., Mousavi, S. M., Emam-djomeh, Z., Khazaei, J. (2007). Prediction of rheological properties of Iranian bread dough from chemical composition of wheat flour by using artificial neural networks. Journal of Food Engineering, 87, 728-734. http://doi.org/10.1016/j. jfoodeng.2007.01.009

Rossi, F., Velázquez, D., Monedero, l., \& Biscarri, F. (2014). Artificial neural networks and physical modeling for determination of baseline consumption of CHP plants. Expert Systems with Applications, 47(10), 4658-4669. http://doi.org/10.1016/j. eswa.2014.02.001

Silva, V. M. Da, Torres Filho, R. D. A., \& Resende, I. V. De. (2018). Rheological properties of selected Brazilian honeys as a function of temperature and soluble solid concentration. International Journal of Food Properties, 2000), s2481-S2494. http://doi.or g/10.1080/10942912.2017.1370599
Silva, V. M. da, de Carvalho, L. A., de Oliveira, N. L., Torres Filho, R. de A., de Resende, J. V. (2016). Rheological and thermal properties of selected Brazilian honeys from various floral origins. Journal of Texture Studies, 47,208-219. http://doi.org/10.1111/jtxs.12174

Soltani, M., Omid, M., \& Alimardani, R. (2014). Egg volume prediction using machine vision technique based on pappus theorem and artificial neural network. Journal of Food Science and Technology, 52(May), 3065-3071. http://doi.org/10.1007/s13197014-1350-6

$X i$, J., Xue, Y., Xu, Y., \& Shen, Y. (2013). Artificial neural network modeling and optimization of ultrahigh pressure extraction of green tea polyphenols. Food Chemistry, 147(1), 320-326. http://doi.org/10.1016/j. foodchem.2013.02.084

Yanniotis, S., Skaltsi, S., \& Karaburnioti, S. (2006). Effect of moisture content on the viscosity of honey at different temperatures. Journal of Food Engineering, 72, 372-377. http://doi.org/10.1016/j. jfoodeng.2004.12.017

Zuluaga-Domínguez, C. M., Nieto-Veloza, A., \& Quicazán-de-Cuenca, M. (2017). Classification of Colombian honeys by electronic nose and physicalchemical parameters, using neural networks and genetic algorithms. Journal of Apicultural Research, 571), 145-152. http://doi.org/10.1080/00218839.2 017.1339521 